\title{
A Prototype Controller for Variable Reluctance Motors
}

\author{
Corrado Guarino Lo Bianco, Alberto Tonielli, and Fabio Filicori
}

\begin{abstract}
A three-level cascade structure is proposed for the control of a variable reluctance (VR) motor. In order to deal with the highly nonlinear behavior of VR motors, the controlling system includes two variable-structure controllers for current and velocity loops as well as an intermediate torque-sharing compensator. The intermediate compensator has been designed by means of nonlinear optimization techniques in order to reduce the torque ripple and to get the maximum motor velocity. The proposed controller has been validated through extensive simulation experiments. The architecture of a prototype controller is presented and the actual performance measured on a VR motor is discussed in comparison with simulations. The results show practical feasibility and good performance of the proposed controller, which is also suitable for a very simple and quite inexpensive fully hardware implementation.
\end{abstract}

\section{NOMENCLATURE}

$v, i, \Phi \quad$ Voltage, current, flux linkage.

$r \quad$ Winding resistance.

$T, T_{L} \quad$ Motor and load torque; $\tilde{T}$ denotes

a particular torque value.

$j \quad$ Phase index.

$\theta, \omega \quad$ Rotor angular position and velocity.

$D, J \quad$ Rotor dumping factor and inertia.

$M \quad$ Number of teeth in the rotor.

$\theta_{1 / 3} \quad$ Angular shift corresponding to one-third of a motor step.

$V_{M} \quad$ Converter feeding voltage.

\section{INTRODUCTION}

$\mathbf{V}$ ARIABLE RELUCTANCE (VR) motors can be designed for very low velocity and very high torque capability, so that they can be used as direct-drive actuators. To meet the specifications required for a direct-drive actuator the motor nonlinearity has to be carefully taken into account through a suitable model. Besides, an advanced control system that linearizes motor and load characteristics must be designed [1]-[4].

The research activity reported in this paper is the natural prosecution of the work presented by the same authors in [1]. Concepts and results already reported in that work are here only referenced and discussed.

Manuscript received October 15, 1994; revised May 22, 1995. This work was supported in part by CIOC-CNR and MURST, National Project on Control Engineering.

The authors are with the Department of Electronics, Computers, and System Sciences, University of Bologna, 40136 Bologna, Italy.

Publisher Item Identifier S 0278-0046(96)01276-2.
This work aims at the prototype implementation of a high performance dynamic controller for a VR motor; this controller is intended for velocity-trajectory tracking in robotic applications.

The design of a velocity controller for a direct-drive VR motor is strongly simplified if a cascade structure is adopted. In addition to advantages deriving from the separation of low-dynamic (velocity) and high-dynamic signals (currents), it was shown in [1] that a cascade structure enables the use of an intermediate torque-sharing control level where the redundancy in the torque generation mechanism of VR motors can be conveniently used for design optimization.

The main design specifications for the proposed controller are robustness, ripple-free torque generation, maximization of velocity at nominal torque and limited voltage, in addition to the feasibility of prototype implementation using off-the-shelf technology.

The proposed solution, even if tuned on a specific VR motor-namely the NSK Motornetics RS-1410 [7]—can be used for other VR or switched reluctance (SR) motors with magnetically decoupled phases, both of rotating and linear types.

The presentation is divided into two sections. In Section II, after a brief presentation of the adopted nonlinear motor model and a general description of the proposed three-level cascade controller, the three control levels are considered in detail. The intermediate torque-sharing feedforward compensator is described first. The optimization procedure used for its design is presented and discussed. A duality property with the optimization procedure proposed in [1] is also shown. The current-tracking controller is then briefly illustrated and the need for a large bandwidth implementation discussed. The robust velocity controller is finally considered. Some properties and the tuning procedure for the motor considered are presented.

In Section III, correct performance of the proposed controller is previously verified by means of extensive simulation experiments. The three cascade controllers are tested separately to verify their specific performance and then together in the overall cascade structure. The prototype controller is then presented in III-B. A hybrid implementation is adopted. Current controllers are fully hardware implemented to get the highest dynamic response at the lowest cost. The other two control levels are implemented via software on a DSP, using a rapid prototyping station [8]. Experimental results are presented in III-C, showing a good agreement with simulations 


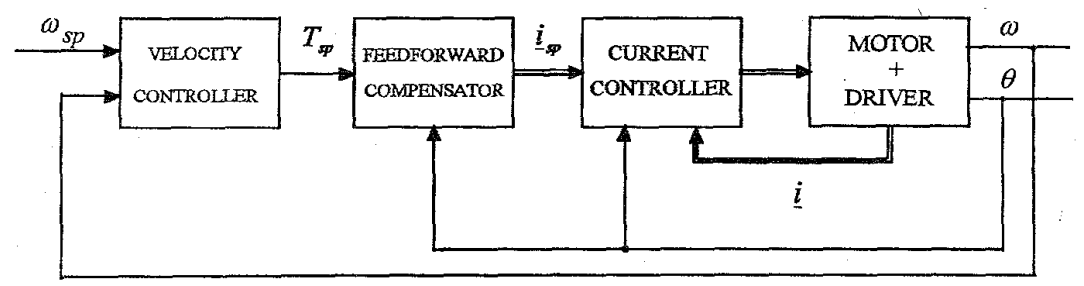

Fig. 1. Motor controller.

and proving the quality and the feasibility of the proposed solution.

\section{CONTROLLER DESIGN}

\section{A. Motor Model and Controller Structure}

The same motor model proposed in [1] is used. The following hypotheses are made:

a) magnetic hysteresis and Foucault currents are negligible;

b) the three phases are almost completely decoupled.

A mechanical equation accounting for variable load torque and inertia is considered.

State Equations:

$$
\frac{d \Phi_{j}}{d t}=-r f\left(\Phi_{j}, \theta_{j}\right)+v_{j} ; \theta_{j}=\theta+j \theta_{1 / 3} ; \quad j=0,1,2
$$

$$
\begin{gathered}
\frac{d \omega}{d t}=-\frac{D+\dot{J}}{J} \omega+\frac{T}{J}-\frac{T_{L}}{J} ; \\
\frac{d \theta}{d t}=\omega .
\end{gathered}
$$

Output Equations:

$$
\begin{gathered}
i_{j}=f\left(\Phi_{j}, \theta_{j}\right)=F\left(\Phi_{j}\right)+R\left(\theta_{j}\right) \Phi_{j} ; j=0,1,2 ; \\
T=-\frac{1}{2} \sum_{j=0}^{2} \frac{d R\left(\theta_{j}\right)}{d \theta_{j}} \Phi_{j}^{2} \triangleq \sum_{j=0}^{2 !} \Phi_{j}^{2} h\left(\theta_{j}\right) .
\end{gathered}
$$

A nonlinear magnetic model is adopted since the motor must be operated under magnetic saturation to maximize the torque/mass ratio. Equation (4) is valid under the hypothesis that the nonlinear function $f(\theta, \Phi)$ can be split into the sum of two simpler functions. $F(\Phi)$ is monotone and accounts for magnetic nonlinearity (saturation). $R(\theta)$ is periodic and accounts for angular nonlinearity.

Although torque equation (5) is similar to that adopted for nonsaturated motors, magnetic saturation is handled. All the effects of magnetic saturation are included in $F(\Phi)$ and the slight dependence of $R(\theta)$ on $\Phi$ is neglected. The experimental measurements reported in [1] show that this approximation is acceptable since there is a good agreement between the model torque and the actual torque, even when strong saturation occurs.
Equation (5) shows that a variable flux must be generated to produce a constant torque in all angular positions.

In [1] it is shown that a large number of harmonics is required to model optimal flux profiles ensuring constant torque in all angular position. Although one of the scopes of this paper is to compute optimal flux profiles with a reduced number of harmonics, a large bandwidth current generation is required to get constant torque.

The main design specifications for the prototype velocity controller are

- very fast current tracking capability;

- optimal use of redundancy in the torque generation mechanism;

- robustness of the velocity controller to handle variable load torque and inertia.

By adopting a cascade control structure, the different control problems can be faced at the different levels using the right methodology and the most suitable technology. The three-level cascade controller reported in Fig. 1 is adopted. It consists of

1) a large bandwidth robust current-tracking controller, designed with hysteresis-type techniques;

2) a static torque-sharing feedforward compensator, designed with optimization techniques;

3) a robust velocity controller, designed with dynamic sliding mode techniques [4], [6].

\section{B. Torque-Sharing Feedforward Compensator}

State equation (1) and torque equation (5) clearly show the redundancy in the torque generation mechanism of VR motors. Three independent phases contribute to generate a scalar torque. An infinite number of different flux vectors, which generate the same torque, exist. If a motor model is available, optimization methods can be used to achieve optimal torque sharing among the three phases. In [12] the function $f(\theta, \Phi)$ was empirically expressed by interpolating matrix data arrays by means of bicubic splines. The same was done for $T(\theta, \Phi)$. That representation, although it well models the motor nonlinearity, does not allow an analytic approach for the optimization problem. In [13] the torque sharing problem was addressed. The degrees of freedom were used to minimize the maximum value of the phase current; in [1], instead, two optimal solutions were found, the first minimizing the power losses and the second minimizing the maximum motor feeding voltage. Apart from the problem considered, an important difference exists between the techniques adopted. in the two papers: In [13] the optimization problem was 
solved empirically while in [1] the problem complexity has required an analytic approach. In [1] it was shown that, to minimize the maximum feeding voltage, it is necessary to feed simultaneously all motor phases (not only two). This requirement strongly complicates the design of the torque sharing compensator. It can be fulfilled only if an analytic model of the motor is available.

The same conceptual approach as that followed in [1] is assumed in this paper with a different optimization criterion. In [1] the feeding voltage was minimized at fixed velocity. In this paper the motor velocity is maximized at fixed feeding voltage. This is motivated by the simple engineering consideration that usually the maximum feeding voltage in an actual drive is not an independent variable. The dc supply voltage of a power converter is usually obtained by rectifying an ac mains and, consequently, has a fixed value.

The proposed torque-sharing compensator is designed to maximize the motor velocity at maximum torque, under the constraints of ripple-free torque and bounded motor feeding voltage. Owing to the strong nonlinearity of the motor equations, a nonlinear optimization procedure is required, which is not suitable for on-line implementation. After some simple algebraic manipulations of torque equation (5), it can be shown that an off-line optimization procedure can be used to design the torque-sharing compensator for a single torque value. In the following the symbol $\tilde{T}$ will be used to represent the value corresponding to the maximum torque that the motor can generate without ripple; this maximum value will be used in the off-line search for the optimal flux profile. Any other lower torque value will be generated on-line, by scaling down the solution achieved for the maximum torque $\tilde{T}$. In the following, the phase suffix $j$ will be omitted since the optimization procedure is applied to a single motor phase.

Let us assume that the optimal flux function $\Phi=\Phi(\tilde{T}, \theta)=$ $\tilde{\Phi}(\theta)$, which generates the maximum torque value $\tilde{T}$, has already been computed.

From (5) it follows that

$$
\tilde{T}=\sum_{j=0}^{2} \tilde{\Phi}^{2}\left(\theta_{j}\right) h\left(\theta_{j}\right)
$$

When a smaller torque (for example $T=K \tilde{T}$ with $0 \leq K \leq 1$ ) is required, (6) can be rewritten as

$$
T=K \tilde{T}=\sum_{j=0}^{2} K \tilde{\Phi}^{2}\left(\theta_{j}\right) h\left(\theta_{j}\right)=\sum_{j=0}^{2} \Phi^{2}\left(\theta_{j}\right) h\left(\theta_{j}\right) .
$$

Comparing (5) and (7), the same torque is generated if

$$
K \tilde{\Phi}^{2}\left(\theta_{j}\right) h\left(\theta_{j}\right) ı \Phi^{2}\left(\theta_{j}\right) h\left(\theta_{j}\right) \quad \forall j=0 \cdots 2
$$

or, equivalently, if

$$
\Phi(\theta)=\sqrt{K} \tilde{\Phi}(\theta)=\sqrt{\frac{T}{\tilde{T}}} \tilde{\Phi}(\theta) .
$$

Actual flux reference signals are obtained by scaling optimal flux $\tilde{\Phi}(\theta)$ according to (9) and by applying the proper angular shifting for the three phases.

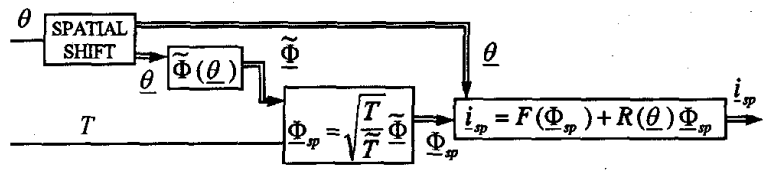

Fig. 2. Block diagram of the torque-sharing compensator.

Implementation of a flux controller would require measurement or estimation of motor fluxes. Both solutions are difficult to implement in an actual drive since flux measurement requires special sensors inside the motor, while flux estimation requires a complex nonlinear algorithm. By using (4), optimal fluxes can be transformed into optimal currents leading to the torque-sharing feedforward compensator illustrated in Fig. 2.

Computation of Optimal Flux: The aim of our optimization is the computation of a single phase flux profile (the remaining two will be obtained by means of an angular shift) such that the maximum possible velocity can be obtained while supplying the maximum torque $\tilde{T}$. The solution must satisfy the following constraints:

a) ripple-free torque,

b) admissible flux levels,

c) bounded motor feeding voltage.

From a mathematical point of view, this is equivalent to solving the following problem

$$
\max _{\Phi, \omega}\{|\omega|\}
$$

with the constraints

$$
\begin{gathered}
\sum_{j=0}^{2} \Phi^{2}\left(\theta+j \theta_{1 / 3}\right) h\left(\theta+j \theta_{1 / 3}\right)=\tilde{T}=\text { const } \\
0 \leq \Phi(\theta) \leq \Phi_{\max } \\
|v(\theta)| \leq V_{M}
\end{gathered}
$$

for all $\theta \in[0,2 \pi / M]$.

Equations (10)-(13) define a functional optimization problem that is linear in the fitness function and nonlinear in the constraints. The problem is first transformed into a semiinfinite optimization [16] and, then, into a sequential quadratic programming (SQP) problem [14], [15]. As a first step, (13) is expressed in terms of the unknown function $\Phi(\theta)$.

By neglecting the small Ohmic voltage drop, the state equation (1) can be rewritten as

$$
v=\frac{d \Phi}{d t}=\frac{\partial \Phi}{\partial \theta} \frac{d \theta}{d t}+\frac{\partial \Phi}{\partial T} \frac{d T}{d t} .
$$

Since optimization is performed at a constant torque, the last term of (14) can be neglected leading to

$$
v \cong \frac{\partial \Phi}{\partial \theta} \omega \text {. }
$$

This is not really a strong restriction, even in actual applications where torque is variable. If the flux derivative with respect torque is bounded, the second term of (14) can be limited by imposing an upper bound for the torque derivative $(\dot{T})$. 
The problem (10)-(13) can also be seen as a min-max problem. Equation (15) allows constraint (13) to be rewritten as

$$
\max _{\theta}=\left\{\left|\frac{\partial \Phi}{\partial \theta}\right|\right\}|\omega| \leq V_{M} ; \quad 0 \leq \theta \leq \frac{2 \pi}{M} .
$$

An explicit relationship between velocity and flux derivative can be derived from (16) as

$$
|\omega| \leq \max _{\theta} \frac{V_{M}}{\left\{\left|\frac{\partial \Phi}{\partial \theta}\right|\right\}} .
$$

Consequently

$$
\max _{\Phi(\theta)}\{|\omega|\}=\max _{\Phi(\theta)}\left\{\max _{\theta} \frac{V_{M}}{\left\{\left|\frac{\partial \Phi}{\partial \theta}\right|\right\}}\right\} .
$$

Thus, the original optimization problem (10)-(13) is equivalent to

$$
\min _{\Phi(\theta)}\left\{\max _{\theta}\left\{\left|\frac{\partial \Phi}{\partial \theta}\right|\right\}\right\}
$$

with constraints (11) and (12).

An interesting duality property with the optimization method proposed in [1] can be demonstrated. The optimization problem solved in [1] was

$$
\min _{\Phi(\theta)}\left\{\max _{\theta}\{|v|\}\right\}
$$

with constraints (11) and (12). By assuming that (15) is a valid representation for the phase feeding voltage and observing that the minimization does not depend on $\omega,(20)$ can be easily rearranged as (19).

In conclusion, it turns out that minimization of the maximum phase voltage and maximization of the rotor velocity are two different aspects of the same problem.

The infinite number of constraints complicates the solution of problem (10)-(13) and of problems (19), (11), and (12) as well. A solution can be directly found by considering a finite number of angular positions, thus reducing the original problem to a classical nonlinear optimization problem with a finite number of constraints. The results presented in [1] show that a large number of angular values must be considered in order to limit the maximum phase voltage and, consequently, a large number of harmonics are required to represent the function $\Phi(\theta)$.

Since the solution technique proposed in this paper also attempts to reduce the number of harmonics, a different approach is considered.

The periodic flux is represented by means of a Fourier series as

$$
\Phi(\theta)=\Phi_{0}+\sum_{i=1}^{L} a_{i} \sin (i M \theta)+b_{i} \cos (i M \theta)
$$

Constraints (11) and (12) are evaluated on a limited number of angular position such that $\theta$ disappears from those equations which become functions of $a_{i}, b_{i}, \Phi_{0}$ only. On the contrary,

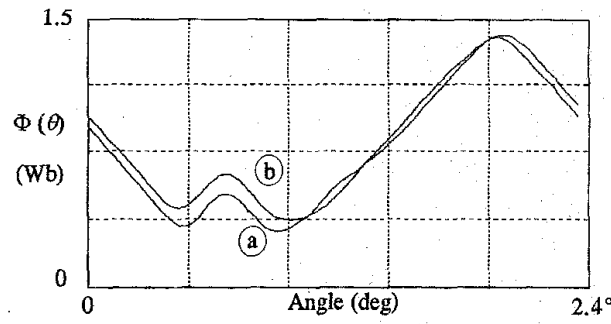

Fig. 3. Waveform (a): Flux function proposed in [1] (13 harmonics); Waveform (b): Flux function adopted in this paper ( 7 harmonics).

constraint (13), being more critical, is left unchanged. The flux derivative with respect to position is obtained from (21)

$$
\frac{\partial \Phi}{\partial \theta}=M \sum_{i=1}^{L} i\left[a_{i} \cos (i M \theta)-b_{i} \sin (i M \theta)\right] .
$$

The original problem is thus transformed into a semi-infinite problem where $a_{i}, b_{i}, \Phi_{0}$ and $\omega$ are the independent variables while $\theta$ is the uncertain parameter.

The solution of problem (10)-(13) is found by modifying a nonlinear programming algorithm of Matlab [9], based on SQP techniques. The semi-infinite programming problem is rearranged to match a classical nonlinear programming problem.

The constraint on the maximum phase voltage can be expressed indifferently by means of (13) or (16). For this reason, every time the SQP algorithm requires the constraints to be evaluated, in order to test the feasibility of the current solution, a Matlab function that solves problem (16) is "called." In this way, variable $\theta$ disappears also from the phase voltage constraint.

Attention must be paid to the solution of problem (16); since $\frac{\partial \Phi}{\partial \theta}$ is not unimodal with respect to $\theta$. In order to overcome local maxima, the searching range is first divided into regular intervals to find an initial, approximate, solution then, starting from that solution, the exact maximum point is obtained by means of a Gauss-Newton algorithm.

By adopting representation (22), the optimization problem (19) becomes

$$
\min _{a_{j}, b_{j}, \Phi_{0}}\left\{\max _{\theta}\left\{\left|\frac{\partial \Phi}{\partial \theta}\right|\right\}\right\}
$$

with the constraints (11) and (12). Constraints (11) and (12) are handled in exactly the same way as in problem (10)-(13).

Also this optimization procedure is implemented by using the optimization tool-box in Matlab [9]. As expected, both algorithms give the same result. The optimal solution proposed here is made up of only 7 harmonics as compared to the 13 resulting from that proposed in [1], with the same residual torque ripple.

The new optimal solution is reported in Table 1 , while in Fig. 3 it is compared with that presented in [1]. It must be pointed out that, with the proposed approach, the optimal flux profile never goes to zero: all phases are active at the same time. This is necessary to get the maximum motor velocity since the less the flux is variable, the less voltage is required to feed the motor. 
TABLE I

Fourier Series CoefFicients of the Optimal Solution

\begin{tabular}{|c|c|}
\hline$a_{j}$ & $b_{j}$ \\
\hline$-4.0575947151882888 \mathrm{e}-001$ & $2.0814118710411036 \mathrm{e}-001$ \\
\hline$-8.0705523813888624 \mathrm{e}-002$ & $-9.4680703446250048 \mathrm{e}-002$ \\
\hline$-4.0622741584571328 \mathrm{e}-002$ & $1.5142289177840746 \mathrm{e}-002$ \\
\hline $4.4220417529617016 \mathrm{e}-002$ & $1.7234685615271240 \mathrm{e}-002$ \\
\hline $1.9235484323677244 \mathrm{e}-002$ & $-1.6096825625186336 \mathrm{e}-002$ \\
\hline$-8.8881050160625536 \mathrm{e}-003$ & $-5.7125133816180936 \mathrm{e}-003$ \\
\hline$-8.7014733387873728 \mathrm{e}-003$ & $8.6851546813071904 \mathrm{e}-003$ \\
\hline \multicolumn{2}{|r}{$\Phi_{0}=8.1096214412877984 \mathrm{e}-001$} \\
\hline
\end{tabular}

By supplying the inverter with a dc voltage of $540 \mathrm{~V}$, a maximum velocity of $6.5 \mathrm{rad} / \mathrm{s}$, is obtained with both optimal flux functions, with a residual torque ripple of $\pm 0.5 \mathrm{Nm}$.

The harmonic content of the optimal flux function has a strong impact on the design of the flux controller. The NSKMotornetics RS-1410 motor has 150 teeth. At a velocity of $6.5 \mathrm{rad} / \mathrm{s}$, the flux fundamental harmonic is at $160 \mathrm{~Hz}$. A bandwidth of at least $1.5 \mathrm{kHz}$ is required to track a flux set-point with 7 harmonics and an almost double one is required for 13 harmonics. Digital implementation of both the flux observer and the controller would require a very short sampling time and a powerful DSP for actual implementation. This suggests the adopted solution, which is based on the transformation of optimal fluxes into equivalent optimal currents through model (4). This prevents the need for an observer, since currents can be easily and inexpensively measured and the current controller is suitable for a simple hardware implementation.

\section{Current Controller}

The large bandwidth of the current signals and the robustness required to compensate for the nonlinearity in motor electrical equation (1), together with the intrinsic switching characteristic of the power converter, suggest the use of an hysteresis current controller for each phase.

If a classical two-level control law $\left(v=+V_{M}\right)$ is adopted, the switching frequency becomes very high. To reduce the switching frequency a simple control law with three output levels is adopted $\left(v=0, v= \pm V_{M}\right)$. The zero voltage output can be very useful to reduce the converter switching frequency.

As reported in Fig. 4, two different hysteresis bands are used, both of them centred with respect to the current set-point. The back-e.m.f. sign can be estimated by observing the current trajectory when the zero-voltage control is applied. Once the back-e.m.f. sign is known, the current can be constrained within the internal band using both a single active control $\left(v=+V_{M}\right.$ or $\left.v=-V_{M}\right)$ and the zero-voltage one. In this way, a strong reduction of the switching frequency is obtained, since the control action and the back-e.m.f. never sum-up. The external hysteresis band is required to recognize a change in the back-e.m.f. direction, as shown in Fig. 4.

For this simple first order scalar system, stability is ensured if enough feeding voltage is available to compensate for back-e.m.f. [4]. The optimal flux profile was computed by

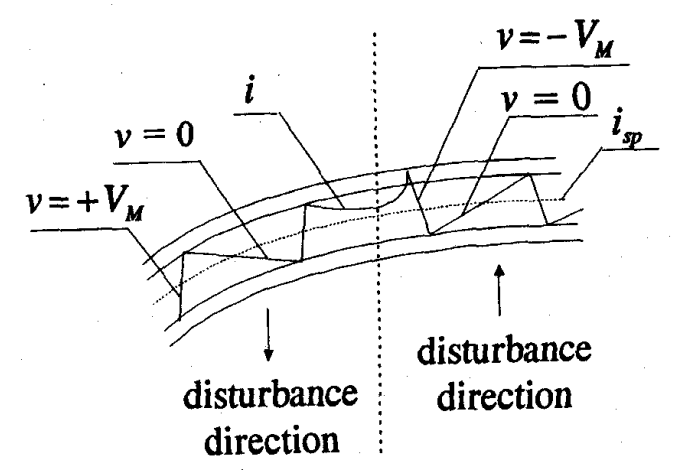

Fig. 4. Hysteresis current control law.

maximizing motor velocity under the constraint of a limited phase voltage. As a result, the system stability is ensured for every motor velocity lower than the maximum value resulting from the optimization procedure.

\section{Velocity Controller}

In robotic applications the motor load is strongly variable, depending on the operating conditions and, generally, unknown. Thus, a robust controller is needed, which is capable of compensating for load torque and for inertia variations, as well as for the disturbances introduced by the modeling inaccuracies and the current off-set generated by the hysteresis controller.

The proposed torque controller has a very fast dynamic response and almost cancels the electrical dynamics. Thus, the velocity tracking controller can be designed on the sole basis of the mechanical equation (2).

The model can be rewritten in error form. In this way, velocity tracking is assured if the system in error form is stable. By defining $\omega_{\text {sp }}$ as the velocity set-point, the velocity error is

$$
\omega_{e}=\omega_{\mathrm{sp}}-\omega
$$

From (2) it follows that the state equation of the system written in error form is

$$
\dot{\omega}_{e}-\frac{D}{J} \omega_{e}-\frac{T_{m}}{J}+\frac{\psi(t)}{J}+\frac{\varphi\left(\omega_{e}, t\right)}{J}
$$

where motor torque is the input variable.

The term $\psi(t)$ collects all exogenous disturbances and is defined as

$$
\psi(t)=(D+\dot{J}) \omega_{\mathrm{sp}}+\dot{\omega}_{\mathrm{sp}} J+T_{L}
$$

The term $\varphi\left(\omega_{e}, t\right)$ indicates the endogenous disturbance produced by the variable load inertia

$$
\varphi\left(\omega_{e}, t\right)=\dot{J} \omega_{e}
$$

The controller must be designed to reach the surface $\omega_{e}=0$ in a finite time $t^{*}$ and to stay on that surface for each $t>t^{*}$.

To stabilize system (25) a discontinuous controller with an integral term is adopted. Originally presented in [6] in a general theoretical framework, this solution was proposed by one of the authors in [4] for velocity control of VR motors in direct drive robotic applications. Mathematical details on the 
stability proof and on robustness properties can be found in [4] and [6]. Here controller equations and design inequalities are reported and briefly discussed, mainly to correlate the controller terms and the related parameters to disturbance characteristics. System inertia is supposed to be slowly variable, thus enabling the effects introduced by $\dot{J}$ to be neglected. This permits simplifying the design of the robust controller since endogenous disturbance is not significant. This is not a limitation since in [6] it was shown that, if the system inertia has large dynamics, some more terms can be included into the velocity controller for compensation.

The three-term control law

$$
T=\lambda \omega_{e}+k \operatorname{sign}\left(\omega_{e}\right)+\int_{-\infty}^{t} h \operatorname{sign}\left(\omega_{e}\right) d t
$$

is adopted, with the following design inequalities

$h>\Delta_{1}=\max _{t}\{|\dot{\psi}(t)|\}=\max _{t}\left\{\left|J \ddot{\omega}_{\mathrm{sp}}+D \dot{\omega}_{\mathrm{sp}}+\dot{T}_{L}\right|\right\}$

$k>0$

$\lambda>\frac{\Delta_{1}^{2}}{k h}(1-\ln 2)$.

This variable structure controller with integral action has several advantages over standard sliding mode (SM) controllers, especially when it is used in velocity tracking applications. The same robustness of SM controllers is obtained here with a limited discontinuous action, whose amplitude $(k>0)$ can be quite freely selected by the designer.

By applying this control law, the equation of the controlled system becomes

$\dot{\omega}_{e}=-\frac{D+\lambda}{J} \omega_{e}-\frac{k \operatorname{sign}\left(\omega_{e}\right)}{J}+\frac{1}{J} \int_{-\infty}^{t} \dot{\psi}(t)-h \operatorname{sign}\left(\omega_{e}\right) d t$.

The meaning of each component of the controller can be easily deduced from (32).

To keep $\dot{\omega}_{e}=0$, the integral term compensates for exogenous disturbance $\psi(t)$. For this reason, as shown by (29), term $h$ must be larger than the maximum derivative of the exogenous disturbance.

Term $k$ cancels residual noise assuring the SM condition on the surface $\omega_{e}=0$. In [6] it has been demonstrated that the introduction of the integral compensator allows the amplitude of term $k$ to be reduced with respect to static SM controllers. It is no longer necessary to assume $k$ larger than the maximum disturbance but it is sufficient that $k>0$. A small $k$ reduces chattering around the surface $\omega_{e}=0$. An appropriate choice of term $\lambda$ assures that the velocity regulator is stable in large. A large $\lambda$ allows the surface $\omega_{e}=0$ to be reached in a short time but it causes some chattering around that surface.

\section{PERFormance EVAluation}

\section{A. Simulation Experiments}

Simulation experiments were performed to check the controller behavior under different operating conditions. The motor RS-1410, designed by NSK Motornetics, was considered. The motor characteristics are reported in Table II. The
TABLE II

CHARACTERISTICS OF THE MOTOR RS-1410

\begin{tabular}{|l|c|}
\hline NO. of rotor teeth & 150 \\
\hline NO. of phases & 3 \\
\hline Winding resistance & $r=3,5 \Omega$ \\
\hline Maximum velocity & $\omega=1$ r.p.s. \\
\hline Rotor inertia & $J=1,0 \mathrm{Kgm}^{2}$ \\
\hline Maximum phase current & $i=7,5 \mathrm{~A}$ \\
\hline Maximum flux & $\Phi=1,4 \mathrm{~Wb}$ \\
\hline Maximum peak torque & $T=400,0 \mathrm{Nm}$ \\
\hline Maximum no-ripple torque & $\widetilde{T}=170,0 \mathrm{Nm}$ \\
\hline
\end{tabular}

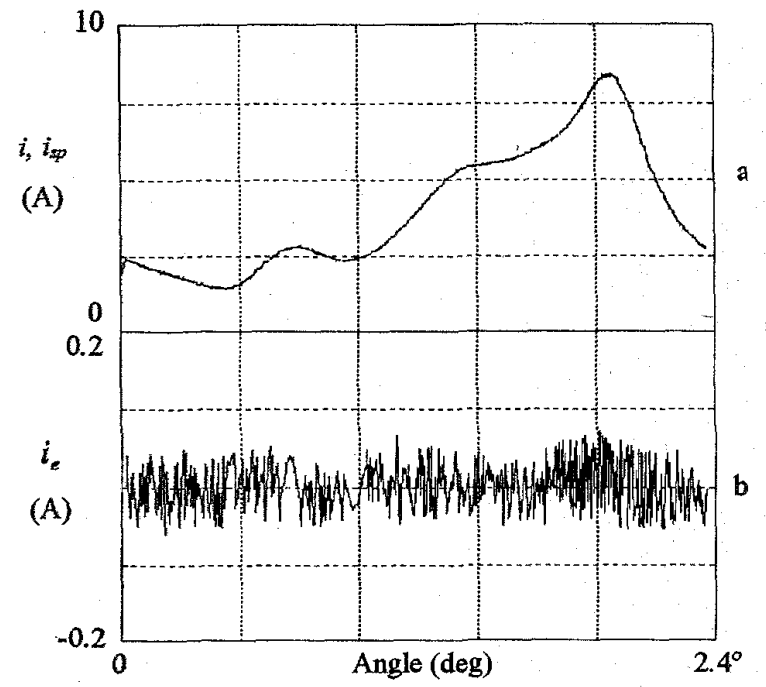

Fig. 5. (a) Current set-point and real current waveforms within a motor step. (b) Current error for a motor step.

effects of the amplitude of the hysteresis band (caused by an upper limitation of the converter switching frequency) on the current controller were considered. The effects of sampling time, computational delay, A/D-D/A converters resolution were verified for the torque-sharing compensator and the velocity controller.

Simulation of the current controller is presented first. The results, shown in Fig. 5, depict the tracking performances of the controller. The amplitude of the internal hysteresis bands is selected at $50 \mathrm{~mA}$. Excellent tracking results for all positions within a motor step. The tracking error is within the expected bounds.

Joint simulations of the torque-sharing compensator and of the current controller have been performed, in order to verify the effects of the computational delay (due to a discretetime implementation of the torque-sharing compensator) and the effects of the hysteresis band amplitude in the current controller. The results are reported in Fig. 6.

All the figures report simulations performed within a motor step with a torque set-point equal to the nominal no-ripple torque. A 50-mA hysteresis band is used except for Fig. 6(d) where a value of $150 \mathrm{~mA}$ is assumed. In Fig. 6(a) the torque 


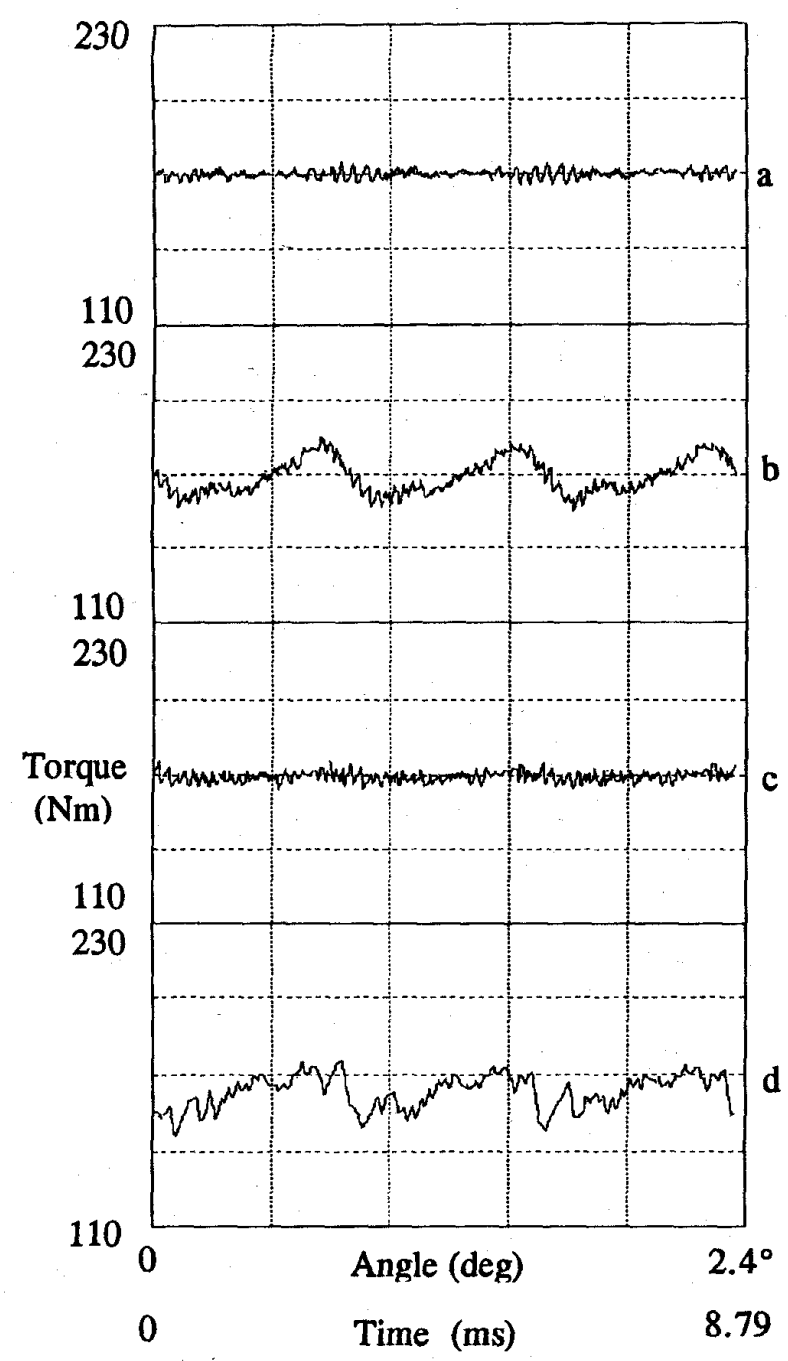

Fig. 6. Simulated torque in a motor step. The motor is rotating at the maximum velocity (a) no computational delay; (b) $100 \mu$ s sampling time; (c) $100 \mu \mathrm{s}$ sampling time with the compensation of the position-error; (d) $100 \mu$ s sampling time with the compensation of the position error and 150 $\mathrm{mA}$ hysteresis region.

generated is reported; no delay is assumed in the optimal flux generation. Ripple-free torque is shown. The residual highfrequency ripple is due to the hysteresis current controller. In Fig. 6(b) a digital implementation of the torque-sharing compensator is assumed, with a sampling time equal to $100 \mu \mathrm{s}$. A larger torque ripple results. The optimal flux $\Phi(\theta, T)$ is a static function of position and torque. Since the torque request made by the velocity controller is constant during the sampling time, the computational delay introduces a position error in the generation of the optimal flux. A partial compensation of this position error can be performed based on the motor velocity.

The effects of the compensation are reported in Fig. 6(c). In Fig. 6(d) the effects of a larger hysteresis band in the current controller are shown. A low-frequency torque ripple arises, mainly due to low-frequency off-set generated by the hysteresis controller.

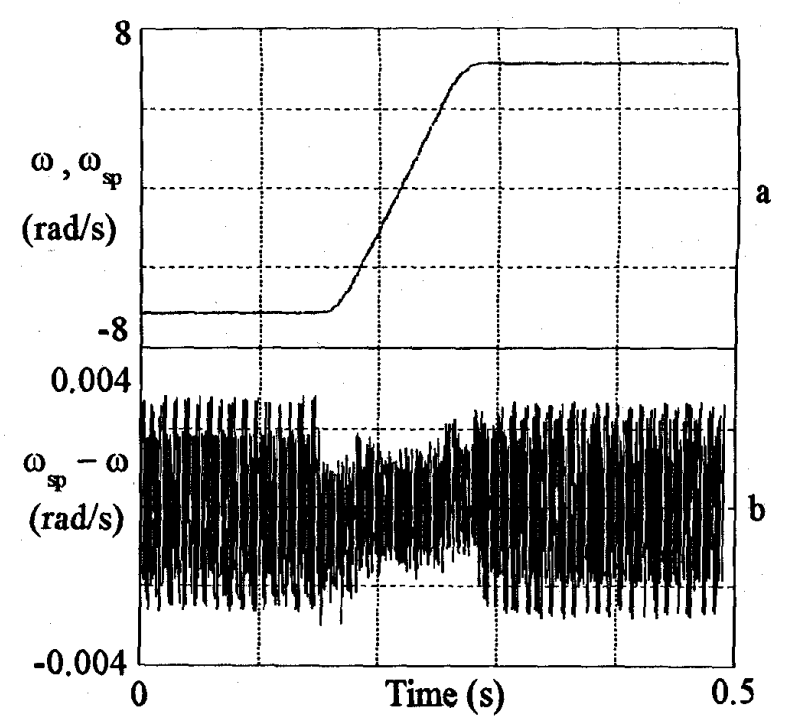

Fig. 7. Motor velocity transient. (a) Velocity set-point and motor velocity. (b) Velocity error.

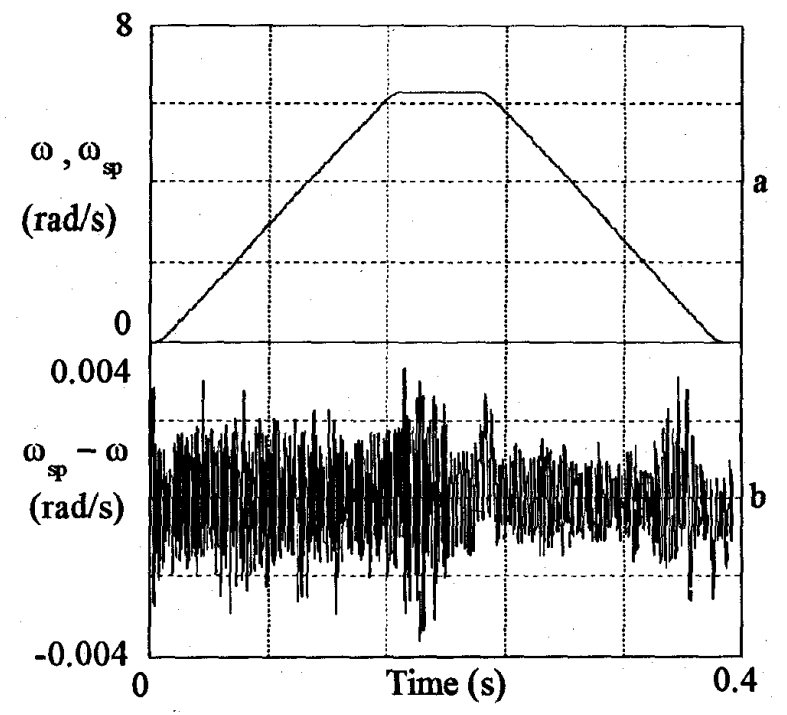

Fig. 8. Motor load inertia variation. (a) Velocity set-point and motor velocity. (b) Velocity error.

According to simulation results, an angular compensation is needed when a discrete-time implementation of the torquesharing compensator is adopted.

Simulations of the velocity controller are reported in Figs. 7 and 8. In Fig. 7 the response to a smooth tracking signal is reported. No external load is connected to the motor. Very good tracking results (Fig. 7(a)), as confirmed by the small tracking error (Fig. 7(b)). The discontinuous term $k$ in the velocity controller, required to ensure robustness, is very small. It leads to a residual velocity ripple less than $1 \%$ of the maximum velocity.

In Fig. 8 a more complex situation is shown. A larger hysteresis band $(150 \mathrm{~mA})$ is adopted for the current controller. A smooth velocity set point is applied at $t=0$ and the motor 


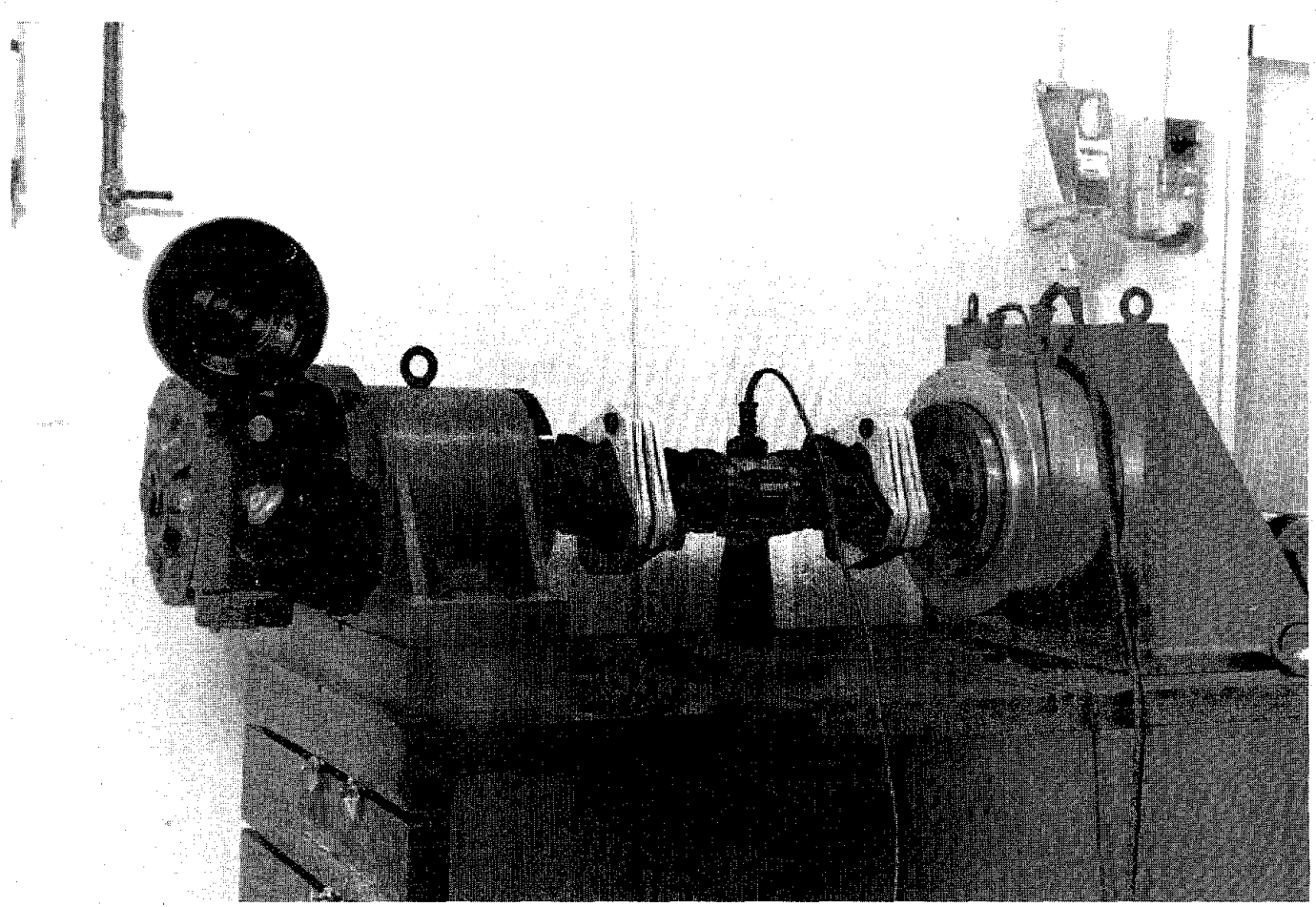

Fig. 9. Test bench for the variable reluctance motor.

is taken at about its maximum velocity. At time $t=200 \mathrm{~ms}$ the load inertia is tripled. At time $t=230 \mathrm{~ms}$, with the motor loaded, the velocity set-point is taken to zero again. Robustness is shown by the two identical transients, the second one performed with a system inertia that is 3 times larger than the first one. The effects of increased inertia are evident from the reduction of the velocity ripple, as reported in Fig. 8(b).

\section{B. Architecture of the Controller Prototype}

From simulation results some interesting considerations must be drawn. Particular care must be taken in the implementation of the current controller. The off-set introduced by the hysteresis band amplitude contributes to increasing the torque ripple. A hardware implementation is preferable, since it ensures very high dynamical response and avoids the delays given by the detection of the hysteresis band crossing, typical of a discrete time implementation. On the other hand, noise in current measurement is typical when a power converter is used. Disturbances in current measurement due to commutations can lead to an incorrect behavior of the hysteresis controller when small values of hysteresis band are selected.

The velocity controller can be implemented via hardware owing to its simplicity, as reported also in [10]. The static torque-sharing compensator can also be implemented by using a look-up table. Nevertheless, it was shown by simulations that, with some design care, a digital implementation of the velocity controller and the torque-sharing compensator gives very good results. The resulting sampling time of $100 \mu \mathrm{s}$ makes possible the implementation of the controller by means of a low cost fixed-point DSP.
To speed up experimentation, the laboratory prototype was designed and built by adopting a hybrid software (velocity and torque-sharing) and hardware (current) implementation. A rapid prototyping station, developed at the Automation and Robotics Lab of the University of Bologna [8], is used for the digital implementation of the velocity and torque-sharing controllers. An especially designed analog/digital board is used to implement the three hysteresis current controllers and to interface the prototyping station with the power converter and with the VR motor.

Rotor position is measured with a Reactasyn sensor, built into the motor. A resolution of more than 150000 pulses per revolution can be obtained in conjunction with a 12-bit resolver to digital converter. Currents are measured by insulated Hall-effect sensors. A 12-bit digital to analog converter is used to interface analog and digital worlds.

Owing to the quadratic characteristics of torque equation (5), only unidirectional load currents are required. Unidirectional currents and three-level output voltage can be obtained by. means of the following switch configurations

$$
\begin{array}{lll}
v=V_{M} & \text { if } & T_{1}=T_{2}=\mathrm{ON} \\
v=-V_{M} & \text { if } & T_{1}=T_{2}=\mathrm{OFF} \\
v=0 & \text { if } & \left\{\begin{array}{l}
T_{1}=\mathrm{ON} ; T_{2}=\mathrm{OFF} \\
T_{1}=\mathrm{OFF} ; T_{2}=\mathrm{ON}
\end{array}\right.
\end{array}
$$

The test bench shown in Fig. 9 was adopted. It is made up of the VR motor (on the right), a connecting shaft including a torquemeter and the load. The load for static measurements consists of a reduction gear with a high reduction ratio $(1 / 3600)$, mainly used to lock or to move the motor to very precise positions. 
TABLE III

VeLocity CONTROLLeR Parameters

\begin{tabular}{|c|c|}
\hline$\lambda$ & $2000 \mathrm{Nm} / \mathrm{rad} / \mathrm{s}$ \\
\hline$h$ & $6000 \mathrm{Nm} / \mathrm{s}$ \\
\hline$k$ & $0.4 \mathrm{Nm}$ \\
\hline $\begin{array}{l}\text { Hysteresis } \\
\text { amplitude }\end{array}$ & $150 \mathrm{~mA}$ \\
\hline
\end{tabular}

\section{Experimental Results}

The values of controller parameters adopted in the experiments are reported in Table III.

Two different experiments are described. The torque controller is tested first. The rotor is locked and the maximum torque that the motor can generate in any rotor position is required to the drive. Very deep magnetic saturation is required to obtain this torque, so that motor nonlinearity (positional and magnetic) is strongly involved.

Using the reduction gear, the motor is then forced to slowly move around a complete motor step. Measured torque is reported in Fig. 10(a). A small amplitude residual torque ripple is detected, lower than $9 \%$ of the torque set-point. Reasonable agreement is obtained between experimental results and the corresponding simulation reported in Fig. 6(d). The high frequency torque ripple is the consequence of current ripple. The low frequency ripple reflects different nonideal effects in the system: a) the off-set generated by the large hysteresis band used in current controllers, in accordance with simulation; b) modeling errors affecting the torque-sharing compensator; c) measurement noise in the torque-meter.

As can be seen from simulation results reported in Fig. 6(c) and (d), a large amount of the torque ripple is due to the amplitude of hysteresis band in the current controller. In our experimental set-up, the amplitude cannot be further reduced owing to some disturbances in current measurement induced by converter switching. Better implementation of the hardware current controller, mainly aimed at reducing these disturbances, is in progress. With a smaller hysteresis band a reduced torque ripple - as confirmed by the simulation reported in Fig. 6(c)-will probably be obtained.

The second experiment is performed in order to test the velocity controller. Velocity transients are in good agreement with the simulated ones. In Fig. 10(b) a velocity inversion with no load is reported. In terms of velocity ripple this is the worst case since inertia is at its lowest value.

Another important result, obtained with this new control technique, is given by the low level of mechanical vibrations, also resulting in a very low acoustic noise generated by the motor. This is due to the combined effect of low torque ripple-which reduces vibrations induced in the overall mechanical structure - and smooth flux variations ensured by optimal flux functions-which reduces vibrations generated inside the motor by the stator windings.

\section{CONCLUSION}

A prototype robust velocity controller for VR motor is presented. Low torque ripple, fast dynamic response, robust-

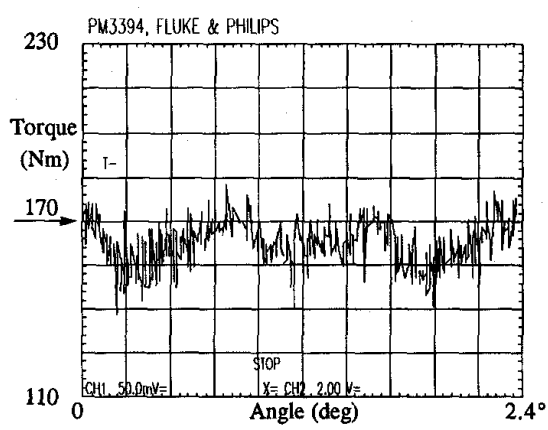

(a)

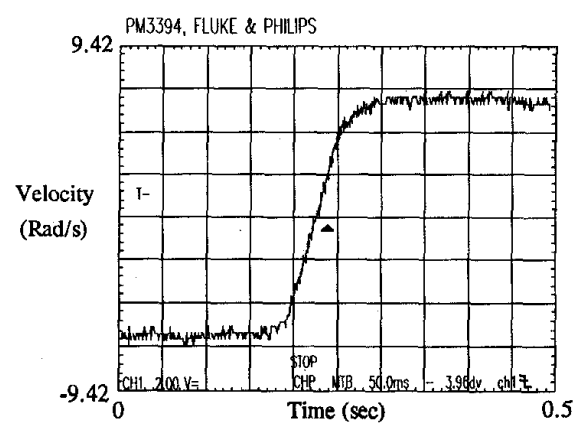

(b)

Fig. 10. Experimental results: (a) Torque measured over a motor step. (b) Motor velocity transient.

ness to parameter variations, and feasibility using off-the-shelf technology are its main characteristics.

Ripple-free torque and smooth optimal flux function have been achieved, thus ensuring a reduced mechanical stress in the motor. This leads to a very low acoustical noise generated by the motor. A rapid prototyping station has been used to implement the discrete-time part of the controller, while a special purpose hardware was designed for hysteresis controllers and I/O interfacing. Simulation results have shown excellent performance for this simple controller. Experimental results confirmed simulations. Some residual torque ripple is experienced, owing to current measurement noise preventing the use of a lower amplitude hysteresis band in the current controllers. A new board, specially designed to reduce the hysteresis band amplitude, is under development. A further reduction in residual torque ripple is expected.

\section{REFERENCES}

[1] F. Filicori, C. Guarino Lo Bianco, and A. Tonielli, "Modeling and control strategies for a variable reluctance direct-drive motor," IEEE Trans. Ind. Electron., vol. 40, no. 1, pp. 105-115, Feb. 1993.

[2] M. Ilic'-Spong, R. Marino, S. M. Peresada, and D. G. Taylor, "Feedback linearizing control of switched reluctance motors," IEEE Trans. Automat. Contr., vol. AC-32, no. 5, pp. 371-379, May 1987.

[3] M. Ilic'-Spong and F. K. Mak, "Torque control of switched reluctance drives with the saturation included," in Incr. Motion Conf., 1986, pp. 275-282.

[4] C. Rossi and A. Tonielli, "Feedback linearizing and sliding mode control of a variable reluctance motor," Int. J. Contr., vol. 60, no. 4, pp. 543-568, Oct. 1994.

[5] D. G. Manzer, M. Varghese, and J. S. Thorp, "Variable reluctance motor characterization," IEEE Trans. Ind. Electron., vol. 36, no. 1, pp. 56-63, Feb. 1989 
[6] R. Zanasi, "Sliding mode using discontinuous control algorithms of integral type," Int. J. Contr., vol. 57, no. 5, pp. 1079-1099, 1993.

[7] Megatorque Motor System, Motornetics Corporation, CA, Dec. 1983.

[8] R. Morici, C. Rossi, and A. Tonielli, "Fast prototyping of nonlinear controllers for electric motor drives," in IFAC World Congress, vol. 2, pp. 445-450, July 1993.

[9] Optimization Toolbox User's Guide, Mathwork Inc., Dec. 1992.

[10] C. Rossi and A. Tonielli, "Robust control of permanent magnet motors: VSS techniques lead to simple hardware implementation," IEEE Trans. Ind Electron.; vol. 41, no. 4, Aug. 1994.

[11] V. I. Utkin, Sliding Modes in Control Optimization. Berlin: SpringerVerlag, 1992.

[12] J. C. Moreira, "Torque ripple minimization in switched reluctance motors via bi-cubic spline interpolation," in PESC '92, June 1992, pp. $851-856$.

[13] D. S. Schramm, B. W. Williams, and T. C. Green, "Torque ripple reduction of switched reluctance motors by phase current optimal profiling," in PESC '92, June 1992, pp. 857-860.

[14] R. Fletcher, "Practical methods of optimization," vol. 2, Constrained Optimization. New York: Wiley, 1980.

[15] P. E. Gill, W. Murray, and M. H. Wright, Practical Optimization. London: Academic Press, 1981.

[16] R. Hettich and K. O. Kortanek, "Semi-infinite programming: Theory, methods and applications," SIAM Rev., vol. 35, no. 3, pp. 380-429, Sept. 1993.

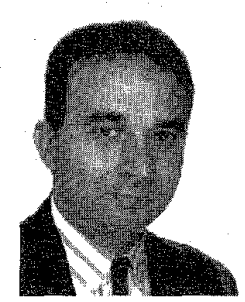

Corrado Guarino Lo Bianco was born in Sassari, Italy, on May 3, 1964. He graduated with honors in electronical engineering in 1989 from University of Bologna, Italy. In 1994 he received the Ph.D. degree from the same university.

His major interests are in the area of digital motor control. In particular, his research activity concerns modeling and control of variable reluctance and asynchronous motors. He is also interested in the application of genetic and interval algorithms in the field of linear and nonlinear control and in the study of the thermal behavior of power converters.

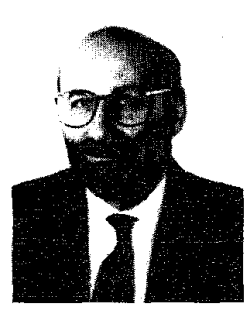

Alberto Tonielli was born in Tossignano, Bologna, Italy, on April 1, 1949. He graduated in electrical engineering from University of Bologna, Italy in 1974.

In 1975 he joined the Department of Electronics, Computer and System Science (DEIS) of the same university with a grant from Ministry of Public Instruction. In 1979 he started teaching Applied Statistics as assistant professor. In 1980 he became permanent researcher. In 1981 he spent two quarters at the University of Florida, Gainesville, as Visiting Associate Professor. Since 1985 he has been an Associate Professor of Control System technologies at the University of Bologna. His main research interests concern digital control, motor drive control and robotics. His current research interests are in the field of nonlinear and sliding mode control for electric motors, non linear observers, robotics, DSP-based control architectures

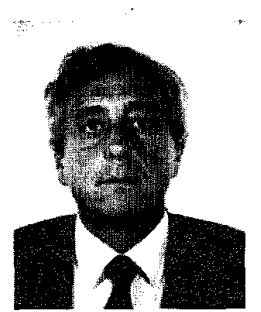

Fabio Filicori was born in Imola, Italy, on January 8,1949 . In 1974 he graduated in electronic engineering at the University of Bologna where, in the same year, he started his academic career at the Istituto di Elettronica as a research assistant in Radio Electronics.

From 1980 to 1989 he was an Associate Professor of Applied Electronics at the same university. In 1990 he became Full Professor of Applied Electronics at the University of Perugia, Italy. The following year he moved to the University of Ferrara, where he was Full Professot from 1991 to 1994. In Nov. 1994 he joined the Dipartimento di Elettronica, Informatica e Sistemistica of the University of Bologna, as a Full Professor of Industrial Electronics. During his academic career he has taught courses on Applied Electronics, Industrial Electronics and Computer-Aided Design of Electronic Circuits. From 1991 he has been President of the Academic Committee for the Degree Course in Electronic Engineering at the University of Ferrara. He is also the Co-ordinator of the Academic Committee for the Ph.D. course in Electronic and Computer Science Engineering at the University of Bologna. His research activity has been mainly devoted to the modeling of electron devices and electronic circuit design, with special emphasis on computer-oriented numerical approaches. In particular, he has been working in the areas of nonlinear circuit analysis and design, electron device modeling, power electronics and digital measurement instruments. 